\title{
Tools for implementing the recent IAU resolutions
}

\author{
George H. Kaplan and John A. Bangert \\ Astronomical Applications Department, U.S. Naval Observatory (USNO), \\ 3450 Massachusetts Avenue NW, Washington, DC 20392-5420, USA \\ email: gkaplan@usno.navy.mil
}

\begin{abstract}
We discuss tools for implementing the recent IAU resolutions, notably the USNO Circular 179 and the NOVAS software package.
\end{abstract}

Keywords. reference systems, astrometry, ephemerides, time, standards

The resolutions on positional astronomy adopted at the 1997 and 2000 IAU General Assemblies are far-reaching in scope, affecting both the details of various computations and the basic concepts upon which they are built. For many scientists and engineers, applying these recommendations to practical problems is thus doubly challenging. Because the U.S. Naval Observatory (USNO) serves a broad base of users, we have provided two different tools to aid in implementing the resolutions, both of which are intended for the person who is knowledgeable but not necessarily expert in positional astronomy. These tools complement the new material that has been added to The Astronomical Almanac, which is prepared jointly by Her Majesty's Nautical Almanac Office (HMNAO) in England and the USNO Astronomical Applications Department (AA). See the paper by Hohenkerk in this Joint Discussion.

USNO Circular 179 is a 118-page book that introduces the recent IAU resolutions to non-specialists. It includes extensive narratives describing the background and basic concepts as well as compilations of the equations necessary to apply the recommendations. The resolutions have been logically grouped into six main chapters: Relativity, Time Scales, The Fundamental Celestial Reference System, Ephemerides of the Major Solar System Bodies, Precession and Nutation, and Modeling the Earth's Rotation. The Circular is available as a hard-cover book or as a PDF file that can be downloaded from the USNO/AA web site <http://aa.usno.navy.mil/ publications/docs/Circular_179.html>. It is also available from <arXiv.org> as [astro-ph/0602086].

NOVAS (Naval Observatory Vector Astrometry Subroutines) is a source-code library available in both Fortran and C. It is a long established package with a wide user base that has recently been extensively revised (in version 3.0) to implement the recent IAU resolutions. However, use of NOVAS does not require detailed knowledge of the resolutions, since commonly requested data - for example, topocentric positions of stars or planets - are provided in a single call to one of the high-level subroutines or functions in the package. There are also low-level routines for individual calculations such as precession, nutation, aberration, sidereal time, etc. NOVAS can be downloaded from the USNO/AA web site <http://aa.usno.navy.mil/software/novas/>.

The two tools are linked, since Circular 179 provides documentation for many of the algorithms used in NOVAS; conversely, NOVAS provides software that implements the formulas given in Circular 179. Both Circular 179 and NOVAS version 3.0 encompass the recommendations of the 2003 - 2006 IAU working groups on precession and nomenclature that were adopted as Resolutions 1 - 3 of the IAU XXVI IAU General Assembly, 2006. 